\title{
Constrained Delaunay Triangulation for Wireless Sensor Networks
}

\author{
Ramnesh Dubey \\ (Department of Computer Science, Nehru Gram Bharati University, India)
}

\begin{abstract}
Wireless Sensor Networks (WSN), an element of pervasive computing, are presently being used on a large scale to monitor real-time environmental status. WSN has the potential of significantly enhancing our ability to monitor and interact with our physical environment. Fault tolerance is one of the main issues in Wireless Sensor Networks (WSNs) since it becomes critical in real deployment environment where reliability and reduced inaccessibility times are important. So, we propose a fault-tolerance technique for coverage area of the sensor network that enhances the energy efficiency by reducing the communication, with the help of Constrained Delaunay Triangulation. Further by applying the above approach, we reduce the energy consumption and congestion in the network. In last, we compared our approach (CDT) with previous approach Delaunay Triangulation (DT) and concluded that our approach is better in fault tolerance and energy saving.
\end{abstract}

Keywords - Wireless sensor networks, Fault-tolerance, Coverage Approach, Energy-efficiency, Event reporting, Delaunay Triangulation, Constrained Delaunay Triangulation.

\section{INTRODUCTION}

Wireless Sensor Networks (WSNs) consists of a large number of tiny sensors used for monitoring, communication, and computational purposes. Sensor nodes are self governing entities that collaborate with each other to perform sensing operations. Their features of self-organization and dynamic reconfiguration make them a perfect choice for applications to monitor and gather physical data in harsh environments [1]. Sensor nodes provide absolute results in monitoring the region of interest.

While sensor nodes have many advantages, they do have some constraints. The tiny size of sensors limits transmission power, bandwidth, and memory space. Also, sensors are energy constrained since they are battery operated. A sensor's primary activities are to sense and to communicate with other nodes to report events to a base station (Sink). The base station processes the data received from sensor nodes and triggers an action for the event monitored. With the constraints possessed by sensors, the following design considerations are essential for better functioning of a sensor network: light weight protocols, reducing the amount of communication, distributed/local pre-computation techniques, complex power saving modes, and large scale networks. Because sensor networks are energy constrained, the primary goal is to maintain energy efficiency of the network [2].

There are several other problems associated with energy efficiency that play a major role in achieving the goals of a deployed sensor network. One such critical problem is coverage. Coverage Approach [3] can be described as how well the geographical region is monitored. Coverage can also be defined as the quality of service provided by a sensor networks. In sensor networks, coverage is classified in several ways based on different criteria. Area coverage [3] is one of the classifications. Area coverage deals with the entire geographical region being monitored, and that every location in the region is monitored by at least one sensor node. Each node monitors an area of geographical region within its boundary, also known as the sensing region and the distance from the node to the boundary is known as the sensing radius. It is essential for a wireless sensor network to monitor every location in the region to provide sensing information, proving the importance of coverage in a sensor network. All locations in geographical region are 1-covered when each location in the region is within the sensing range of at least one sensor node.

Sensor nodes deployed in harsh environments are error prone due to noise interference, and obstacles in the geographical region and terrain. Deployment of sensors providing 1-coverage to handle the challenges posed by the errors in the network is inadequate as they lead to failures in event detection and reduction in quality of service provided by sensors. Fault tolerant mechanisms are essential to handle the error prone nature of a sensor network. K-coverage mechanisms were proposed to provide fault tolerance with degree $\mathrm{K}$. in this section, we address the problem of constructing a connected sensor K-cover, wherein each point in the query region is covered by at least $\mathrm{K}$ distinct sensors or A geographical region is $\mathrm{K}$-covered, provided every point in the region is within the sensing region of $\mathrm{K}$ distinct sensors [3]. To solve the coverage problem we have used Constrained Delaunay Triangulations (CDT).

Delaunay Triangulations (DT) and CDT, they have been very influential in solving the coverage problems of wireless sensor networks. DT and CDT are popular tools used for the representation of planar 
domains. The CDT is an extension of the DT to handle constraints. A CDT can be seen as the triangulation closest to the DT that respects given constraints. Since the Delaunay triangulation is unique for any point set (with the exception of sets with co-circular points), the constrained Delaunay triangulation will most likely contain some edges which are not Delaunay [4,5].

The rest of this paper is organized as follows: In section 2 we present related work 3 we describe Coverage approach in used Constrained Delaunay Triangulation for better in fault tolerance and energy saving. Section 4 we describe our Implementation status. Section 5 concludes our paper.

\section{RELATED WORK}

In WSNs, the coverage problem was initially reviewed as an area coverage problem. As wireless sensor networks are resource constrained, and to provide quality monitoring services, energy efficiency and event reporting play a very important role and contribute to coverage protocols in WSNs. many protocols and Coverage approaches have been proposed to provide coverage which is shown on the Table 1 [6], energy efficiency, and reliable event transfer in WSN research.

Table 1 Area Coverage Approaches

\begin{tabular}{|c|c|c|c|c|c|c|c|}
\hline & & & & $\begin{array}{l}\text { Sensing } \\
\text { Commun } \\
\text { Range R }\end{array}$ & $\begin{array}{l}\text { ange Rs } \\
\text { ication }\end{array}$ & & \\
\hline $\begin{array}{l}\text { Coverage } \\
\text { Approach }\end{array}$ & $\begin{array}{l}\text { Coverage } \\
\text { Type }\end{array}$ & $\begin{array}{l}\text { Problem } \\
\text { Objective }\end{array}$ & $\begin{array}{l}\text { Sensor } \\
\text { Deploymen } \\
\text { t Method }\end{array}$ & $\begin{array}{l}\text { Same } \\
\text { Rs for } \\
\text { all } \\
\text { Sensors } \\
?\end{array}$ & $\begin{array}{l}\text { Is Rs=Rc } \\
\text { for each } \\
\text { Sensors? }\end{array}$ & $\begin{array}{l}\text { Algorith } \\
\mathrm{m} \\
\text { Character } \\
\text { istics }\end{array}$ & $\begin{array}{l}\text { Ref } \\
\text {. }\end{array}$ \\
\hline $\begin{array}{l}\text { Most } \\
\text { constraine } \\
\text { d } \\
\text { minimally } \\
\text { constraini } \\
\text { ng } \\
\text { Heuristic }\end{array}$ & $\begin{array}{l}\text { Area } \\
\text { coverage }\end{array}$ & $\begin{array}{l}\text { Energy } \\
\text { efficiency; } \\
\text { maximize } \\
\text { network } \\
\text { lifetime by } \\
\text { reducing } \\
\text { number of } \\
\text { working } \\
\text { nodes }\end{array}$ & Random & Yes & NA & $\begin{array}{l}\text { Centraliz } \\
\text { ed }\end{array}$ & [2] \\
\hline $\begin{array}{l}\text { Disjoint } \\
\text { dominatin } \\
\text { g sets } \\
\text { heuristic }\end{array}$ & $\begin{array}{l}\text { Area } \\
\text { coverage }\end{array}$ & “ & Random & Yes & NA & $\begin{array}{l}\text { Centraliz } \\
\text { ed }\end{array}$ & {$[1]$} \\
\hline $\begin{array}{l}\text { Node self- } \\
\text { schedulin } \\
\text { g } \\
\text { algorithm }\end{array}$ & $\begin{array}{l}\text { Area } \\
\text { coverage }\end{array}$ & “ & Random & $\begin{array}{l}\text { Yes + } \\
\text { No } \\
\text { (both) }\end{array}$ & NA & $\begin{array}{l}\text { Distribute } \\
\text { d, } \\
\text { localized }\end{array}$ & [8] \\
\hline $\begin{array}{l}\text { Probing- } \\
\text { based } \\
\text { density } \\
\text { control } \\
\text { algorithm }\end{array}$ & $\begin{array}{l}\text { Area } \\
\text { coverage }\end{array}$ & \begin{tabular}{|l} 
Energy \\
efficiency; \\
maximize \\
network \\
lifetime by \\
controllin \\
g working \\
node \\
density
\end{tabular} & Random & Yes & NA & $\begin{array}{l}\text { Distribute } \\
\text { d, } \\
\text { localized }\end{array}$ & [9] \\
\hline
\end{tabular}


Current coverage mechanisms proposed so far do not facilitate fault tolerance and energy efficiency together. Sensor networks are energy constrained as they are battery operated, but in addition to providing fault tolerant coverage, the energy efficiency of the network must be maintained. K-coverage mechanisms proposed in the literature [3] are not energy efficient as several sensors report simultaneously, leading to excessive energy consumption, congestion, and collisions in the network. This reduces the quality of service and network performance.

Shown on table 1.Coverage mechanisms introduced previously only meet the requirement of sensors covering the region of interest within the sensing region of sensor nodes. Current techniques proposed to date have addressed the issue of the area being constantly covered. however, these techniques have failed to address the quality of service in sensor networks, to provide quality of service in monitoring a given region, with the region completely covered, sensors must also detect the events occurring in the region and report them, for improving the quality of service provided by the coverage mechanisms, there is a need for coverage techniques that ensure event detection and reporting.

Coverage problems have been approached in different directions with different constraints and parameters. Shown on table 1 all of the approaches proposed so far are either not energy efficient or do not provide efficient mechanisms of event reporting. The approach which takes care of all the factors is Constrained Delaunay triangulation, which is more efficient than Delaunay triangulation as it covers more coverage area and provides better energy efficiency and event reporting [7].

\section{Describe Coverage Approach In Used Constrained Delaunay Triangulation}

Use Constrained Delaunay Triangulation for better in fault tolerance and energy saving. a constrained Delaunay triangulation is a generalization of the Delaunay triangulation that forces certain required segments into the triangulation. Because a Delaunay triangulation is almost always unique, often a constrained Delaunay triangulation contains edges, thus a constrained Delaunay triangulation often is not a Delaunay triangulation itself.

We chose the distributed greedy heuristic provided in to identify the minimal subset, as it caters to cover the entire region, maintains connectivity between sensor nodes, and also performs better in comparison with other coverage mechanisms proposed.

To improve energy efficiency of the network while maintaining fault tolerance from the subset of 2coverage nodes previously chosen, the subset is further divided into 1-coverage nodes and backup nodes. Backup nodes provide additional support to the 1-coverage nodes in event detection and maintain fault tolerance. Backup nodes improve energy efficiency by reducing the communication as they only report when 1coverage nodes fail to detect the event [3].

The selection process of sub setting of nodes is performed in different stages as part of the preprocessing of WSN to cater quality monitoring services.

In the first stage, we chose the subset $\mathrm{D}$ containing nodes providing 2-coverage that is each and every location is monitored by at least two nodes. In stage two, we use the properties of Constrained Delaunay triangulation and perform a local Constrained Delaunay triangulation over the chosen subset $\mathrm{D}$ providing two coverage. In the final stage, we further divide subset D into two subsets with the knowledge obtained from Constrained Delaunay triangulation in stage two. One subset provides 1-coverage and the other subset provides additional support or backup. Details of how the selection process is performed are presented in further sections below.

Considering a set of $\mathrm{S}$ nodes in a given region, choosing the set $\mathrm{D}$ of minimum number of nodes, providing 2-coverage from $\mathrm{S}$ can be represented as below:

$$
\mathrm{D} \subseteq \mathrm{S}
$$
below.

Further dividing the set $\mathrm{D}$ into sets $\mathrm{A}$ and $\mathrm{B}$, providing 1-coverage nodes and Backup nodes can be shown as

$$
\begin{aligned}
& \mathrm{A} \subseteq \mathrm{D} \\
& \mathrm{B} \subseteq \mathrm{D}
\end{aligned}
$$

$$
\mathrm{A} \cup \mathrm{B} \equiv \mathrm{D}
$$

\subsection{Selection of 2-coverage subset nodes}

Considering an initial set of sensor nodes $\mathrm{S}$ in a given region, a subset of nodes providing 2-coverage is chosen. The selection of a minimum number of sensors from a set $\mathrm{S}$ to provide 2-coverage for a given region is NP-hard, as mentioned before. to select the minimal number of nodes providing 2-coverage, we used the distributed greedy technique for K-coverage proposed in and adapted it to provide 2-coverage. In the distributed greedy heuristic, a minimal number of nodes are selected from the deployed set. Initially, a random node, say A, 
is chosen from the deployed set $\mathrm{S}$ and is identified as 2-coverage node. A now Broadcasts a control message NODE-DBL-STATUS to its one-hop neighbors to select the potential 2-coverage node. The NODE-DBLSTATUS control message is used to query the one-hop neighbors if they are previously chosen as 2-coverage nodes. Upon receiving the NODE-DBL-STATUS message, the one-hop neighbors' reply to the message received from A with a control message YES/NO. the nodes notify A with YES if they have been previously chosen and NO if not chosen. Each and every node replies to the YES/NO control message three times to essentially make sure at least one of the control messages would make it to the node if other control message are dropped due to collisions [3].

To identify a potential 2-coverage node, A performs a computation over the received reply of YES control messages. In this computation, the source node tries to identify the potential 2-coverage node of maximum benefit. The maximum benefit function provided in is a generalized solution for K-coverage. We adapted [3] this approach and found the maximum benefit for 2-coverage. The maximum benefit is calculated based on the maximum overlapped area from the neighboring nodes so as to provide 2-coverage. Once the potential 2-coverage node is chosen from the maximum benefit computation, a sends a control message DBLSTATUS-NOTIFY to notify the identified node as a 2-coverage node. This process continues until the entire geographic region is covered, explained with the help of a pseudo code below. The above procedure is chosen for identifying the subset providing 2-coverage as it ensures the entire region is 2-covered. It also maintains the one-hop connectivity between the sensor nodes in the network so that the nodes can transmit messages and report events to the base station. Once the entire region is covered, the chosen 2-coverage sensor nodes are active and are involved in the sensing and communication activity of the network. The remaining nodes are inactive nodes.

Algorithm 1: Distributed Greedy Algorithm [3]

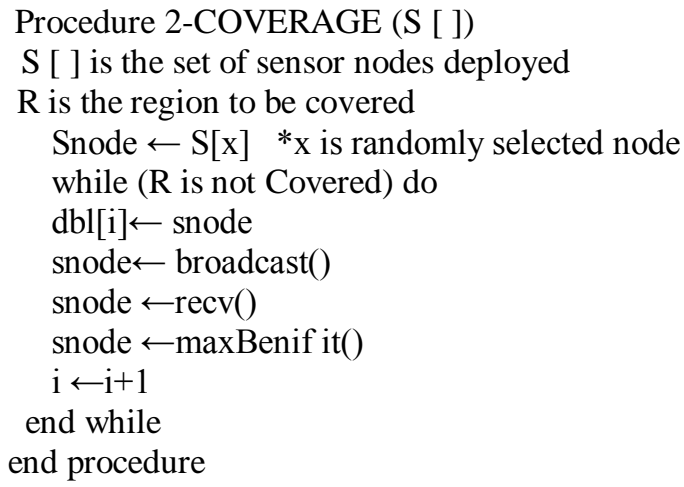

\subsection{Selection of 1-coverage subset and backup nodes from selected 2-coverage subset}

Backup nodes are selected after finding the 2-coverage nodes and the Constrained Delaunay triangulation over a 2-coverage subset. Identification of backup nodes is performed in two stages. Each and every node identifies itself as a backup node if the region it covers is covered entirely by its triangle neighbors, which are not previously chosen as backup nodes.

To illustrate the backup node selection, in Figure 2, node A sends a query control message NODEPRIMARY-STATUS to all of its one-hop neighbors B, C, D, E, and I. the one-hop neighbors check their status and reply to node A if they were previously chosen as primary (1-coverage) nodes or not. Upon receiving reply control messages NODE-PRIMARYSTATUS- REPLY from one-hop neighbors B, C, D, E, and I, node A checks if the nodes that replied are present in the triangulation in which node A is a vertex. In this illustration, nodes B, C, D, and E are Delaunay neighbors in which node A is also part of the triangles. node A computes if it is a valid backup node by checking if the region it covers by itself is completely covered by the Delaunay neighbors. In the set of Delaunay neighbors, if node D is a backup node, then it is not considered in the computation.

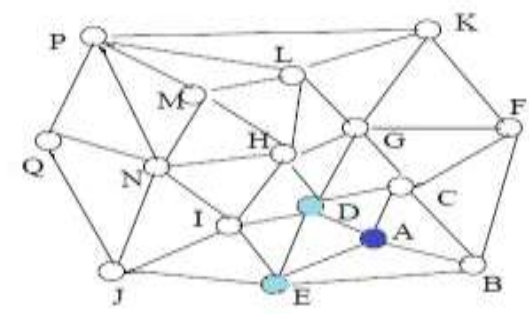

Figure 1 Selection of Backup node from Double Coverage set Node A Backup, Node D, E Primary (1- overage) 
Only non-backup nodes are considered for computation. If the area is completely covered, then node A sets itself as a backup. Once a node is identified as a backup node, it sends a notification control message NODEPRIMARY-STATUS-NOTIFY to the nearest and median distant neighbors, which are $\mathrm{D}$ and $\mathrm{E}$ in the illustration. To provide a better selection of 1-coverage nodes in the topology, nearest and median nodes are chosen. Nodes receiving the NODEPRIMARY- STATUS-NOTIFY message will identify themselves as primary nodes providing 1-coverage. Nodes receiving the NODE-PRIMARY-STATUS-NOTIFY notification message would ignore the message if the node was previously identified as either a backup node or primary node. This process is performed in all nodes to identify backup and primary nodes. All the above processes are performed in stage one.

To reduce the redundancy from primary nodes, backup nodes are again identified based on the same guidelines in stage two. Considering node D as the primary node, it broadcasts a NODE-PRIMARY-STATUS message. upon receiving replies from neighbors A, C, E, G, H, and I, primary node D computes the area covered by itself and the area covered by the primary nodes C, E, G, H, and I, which have replied to node D's NODE-PRIMARY-STATUS message. If the primary nodes $\mathrm{C}, \mathrm{E}, \mathrm{G}, \mathrm{H}$, and I cover the region covered by node $\mathrm{D}$, then node D identifies itself as backup node. For illustration purposes, C and $\mathrm{E}$ are considered primary nodes and $\mathrm{A}$ as backup node in stage 2 . The procedure of selection for backup nodes is also presented in the form of an algorithm [2, 6].

Algorithm 2: Selection of Backup Nodes

Procedure: BK SELECT (dbl [ ])

$\mathrm{dbl}[\mathrm{]}$ is the set of sensor nodes providing 2Coverage

Neighbors [ ] is the set of Triangle Neighbors of each node

$\mathrm{i} \leftarrow 0$

While $\mathrm{i}$ \# dbl.end() do

If $\mathrm{dbl}[\mathrm{i}] \cdot \operatorname{area}() \equiv$ Neighbors [ ].area() then

Backup $[\mathrm{j}] \leftarrow \mathrm{dbl}[\mathrm{i}]$

PotPri[] $\longleftarrow$ nearest(Neighbors[],backup[ j])

PotPri[] $\longleftarrow$ median(Neighbors[], backup[j])

$\mathrm{i} \leftarrow \mathrm{i}+1$

end if

end while

while $\mathrm{i} \neq$ PotPri.end() do

if PotPri.area( $) \equiv$ Neighbors [ ].area( $)$ then

backup[] $\leftarrow$ PotPri[i]

erase(PotPri[i])

end if

end while

end procedure

\subsection{Backup Node Functionality}

For sensor nodes monitoring in harsh environments, several events go undetected due to noise interference, terrain, signal fading, obstacles, and etc. In order to provide additional support, backup nodes assist deployed 1-coverage nodes in detecting the event that occurred in a region. To illustrate the backup node functionality, we represent the network in Figure 2. Circles with solid boundaries are nodes providing 1coverage, circles with dashed boundaries are backup nodes, and BS is base station.

Backup nodes support 1-coverage nodes in improving the fault tolerance of the network by detecting events simultaneously with 1-coverage nodes in the network and reporting the event detected.

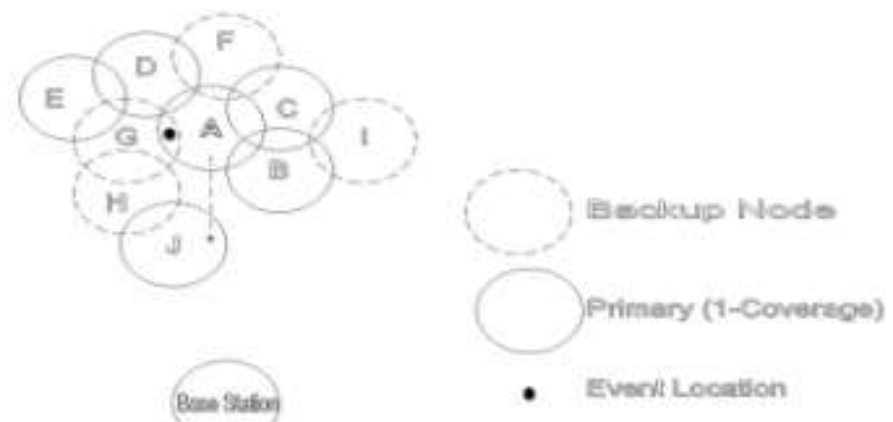

Figure 2 Backup Functionality 
When they know that the 1-coverage neighbors failed to detect the event. In the current literature, coverage protocols have assumed that all the events are successfully detected without considering the error-prone nature of the network. When an event is detected, 1-coverage nodes transmit messages to its forwarder to report the event to the base station. Backup nodes observe the packet transmissions for a time of td, which is the transmit time of a packet for one-hop to determine if the event was successfully detected by 1-coverage nodes. Backup nodes can overhear the packet transmissions, which is used to determine if the event was successfully detected. When 1-coverage nodes do not transmit packets for the event detection within the time $t_{d}$, the backup nodes classify the event detection as unsuccessful and transmit packets to its forwarder to report the event to the base station.

To illustrate the process of event detection in the WSN from Figure 2, consider that an event has occurred at location " 6 " Node A and G have the event within their sensing region, and sense the event. Node A transmits packets to node $\mathrm{J}$ for reporting the event to the base station. Node $\mathrm{J}$ is the forwarder for node $\mathrm{A}$, and forwards the packet received from A to the base station. Node G, as a backup node, will observe for a time $t_{d \mathbb{A}}$ to overhear the packet transmission from node A. Node G, upon overhearing the transmission from node A, considers the event to be successfully detected. If an unsuccessful detection occurs, $G$ would transmit the packet to its forwarder and report the event to base station.

To better illustrate event reporting in a WSN, in Figure 2, an event occurring at location 'G', sensor nodes $\mathrm{A}, \mathrm{B}$, and $\mathrm{C}$ detect the event. Node $\mathrm{B}$, being the nearest node to the base station, reports first. Node $\mathrm{C}$ and Node A report based on time in an alternate path with another forwarder, if nodes $\mathrm{A}$ and $\mathrm{C}$ do not find an alternate forwarder to forward data, they forward data to the backup node to report the event to the base station.

\section{Implementation Status}

We have implemented our Constrained Delaunay Triangulation Strategy, Simulation parameters are shown in the Table 2 and comparison results are shown in Figure 3, 5.

Table 2 Simulation Environment

\begin{tabular}{|l|l|l|}
\hline S.NO. & Features & $\begin{array}{l}\text { Constrained Delaunay Triangulation } \\
\text { Coverage strategy }\end{array}$ \\
\hline 1 & Simulation Scenario & Matlab \\
\hline 2 & Numbers of Nodes & 50 \\
\hline 3 & Area & $1000 \times 1000$ \\
\hline 4 & Dimensions & $2 \mathrm{D}$ \\
\hline 5 & Distance Computed Formula & $\sqrt{\left(x_{2}-x_{1}\right)^{2}+\left(y_{2}-y_{1}\right)^{2}}$ \\
\hline 6 & Sensor Communicate Condition & Distance \\
\hline 7 & Overlapping of Sensing Area & Minimum \\
\hline 8 & Coverage & Area Coverage \\
\hline 9 & Sensing Range & Regular Sensing Range \\
\hline 10 & Strategy & Geometry Based \\
\hline
\end{tabular}

As shown in Figure 5 all nodes are connected as compared to figure 3 where one node is not connected. This is because we have used the area coverage approach where sensor nodes are connected according to Kcover approach, where in each point in the query region is covered by at least $\mathrm{K}$ distinct sensors. Each sensor I is stationary and is associated with a fixed sensing region which is assumed to be a disk of radius SI . Also, each sensor can directly communicate with some of the sensors around it, based on a fixed communication graph and cover the node 42 by Constrained Delaunay Triangulation coverage Strategy. 


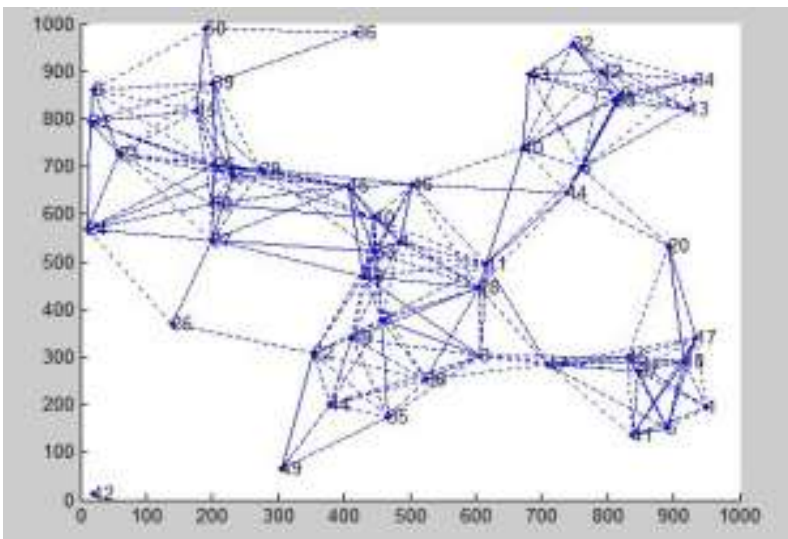

Figure 3 Matlab Simulation for Delaunay Triangulation Coverage Strategy

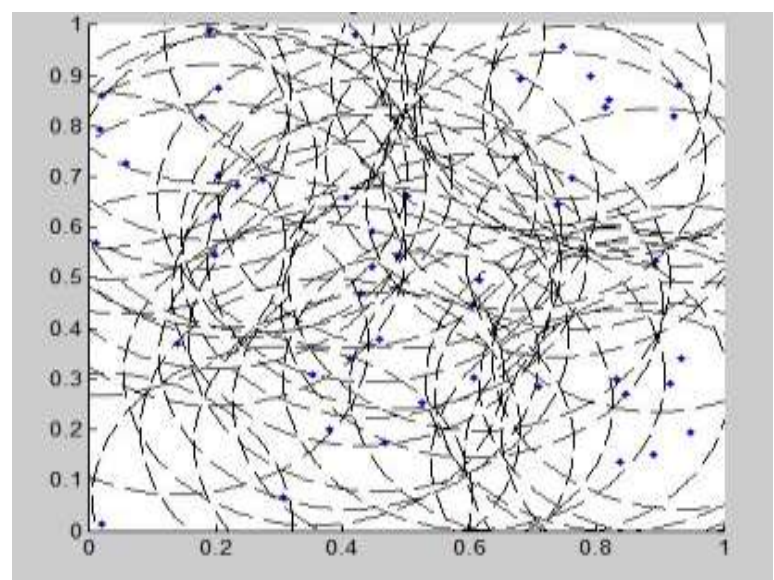

Figure 4 Matlab Simulation for Area Coverage Constrained Delaunay Triangulation Coverage Strategy

We ultimately wish to use constrained Delaunay triangulations that we want to be able to compute a modified version of the Delaunay triangulation in which user-defined edges or "constraints" may be specified. Since the Delaunay triangulation is unique for any point set (with the exception of sets with co-circular points), the constrained Delaunay triangulation will most likely contain some edges which are not Delaunay Figure 4. Constrained Delaunay Triangulation coverage strategies are adopted in finding a maximum number of set covers to monitor the area and provide coverage. The set covers are formed based on the energy levels of each node, its neighbors, and the contribution of the node in sensing Area to provide coverage. Every sensor is added to the set cover incrementally based on the contribution parameter of each node. A sensor that has more detection is given preference for selection in the set cover to provide coverage. Selection of the sensor node into a set cover is repeated to maintain Area coverage all the time. The goal of it is to increase the network lifetime and reduce the energy consumption in addition to providing Area coverage.

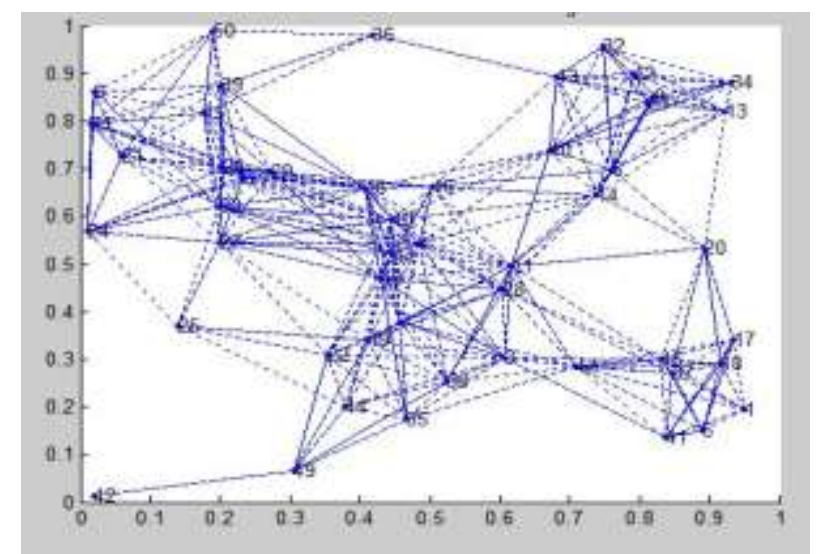

Figure 5 Matlab Simulation for Area Coverage Constrained Delaunay Triangulation Coverage Strategy 


\section{CONCLUSION}

Wireless Sensor Networks are mainly deployed in harsh environments to provide quality services. In such environments, errors in WSN like noise interference, terrain, and obstacles pose problems in detecting the event and thereby degrade the event detection capability of the network. There is a need to provide fault tolerance to detect the events occurring in the geographical region. With current fault tolerant mechanisms, many nodes detect the same event and forward data to the base station, which increases the number of transmissions and congestion in the network. With an increase in the number of transmissions, the energy consumption of nodes increases and the event reporting capability due to collisions in the network is reduced. A decrease in the number of events reported to the base station reduces the quality of service provided by the coverage protocol. Also, as sensor nodes are energy constrained, maintaining energy efficiency is one of the primary concerns of sensor networks.

So to provide quality service by coverage protocols, there arises a need for developing protocols to provide fault tolerance, event reporting, and maintain energy efficiency using Constrained Delaunay Triangulations.

In the future, we would like to investigate for better mechanisms in choosing the minimal number of nodes for our coverage-based protocol and increase lifetime of the sensor network for using routing approach. This way will enrich in the set of nodes chosen, thereby lowering the contention in the network and perform better event reporting operations, with low latency.

\section{References}

[1] M. Cardei, D. MacCallum, X. Cheng, M. Min, X. Jia, D. Li, and D.Z. Du, Wireless sensor networks with energy efficient organization, J. Interconnection Networks, 3, pp. 213-229, 2002

[2] S. Slijepcevic and M. Potkonjak, Power efficient organization of wireless sensor networks, IEEE Int. Conf. Commun., 2, pp. 472476, 2001

[3] Z. Zhou, S. Das, and H. Gupta, Connected k-coverage problem in sensor networks, in Proc. Int. Conf. Computer Communications and Networks (ICCCN), pp. 373-378, 2004

[4] M. Kallmann, H. Bieri, and D. Thalmann. Fully dynamic constrained delaunay triangulations. Geometric Modelling for Scientific Visualization, 2003

[5] R.Dubey, S.K.Swain, R.Bera, C.P.Kashayap, Fault Tolerance in Wireless Sensor Networks using Constrained Delaunay Tringulation, IRnet, ICEECS,.pp.172-178, 2012

[6] M. Cardei, J. Wu, Hand book of Sensor Networks: compact wireless and wired sensing systems, M.Ilyas, I.Mahgoub, (CRC Press , New York, London, Boca Raton, 2005), pp. 361-372.

[7] P. Kumari, Y.Singh, Delaunay Triangulation Coverage Strategy for Wireless Sensor Netowrks, IEEE, ICCCI, pp.1-5, 2012

[8] D. Tian and N.D. Georganas, A coverage-preserving node scheduling scheme for large wireless sensor networks, 1st ACM Workshop Wireless Sensor Networks and Applications, pp. 32-41, 2002

[9] F. Ye, G. Zhong, S. Lu, and L. Zhang, Energy efficient robust sensing coverage in large sensor Networks, Technical report, UCLA, 2002. 